\title{
How do electrons communicate about time?
}

\section{A.L. Godunov ${ }^{\mathrm{a}}$ J.H. McGuire ${ }^{\mathrm{a}}$ S.G. Tolmanov ${ }^{\mathrm{a}}$ Kh. Kh. Shakov ${ }^{\mathrm{a}}$ R. Dörner ${ }^{\mathrm{b}}$ H. Schmidt-Böcking ${ }^{\mathrm{c}}$ R.M. Dreizler ${ }^{\mathrm{d}}$}

\author{
a Department of Physics, Tulane University, New Orleans, LA 70118-5698 \\ ${ }^{b}$ Fakultät für Physik, Universität Freiburg, 79104 Freiburg, Germany \\ ${ }^{c}$ Institut für Kernphysik, Universität Frankfurt, 60486 Frankfurt, Germany \\ ${ }^{d}$ Institut für Theoretische Physik, Universität Frankfurt, 60054 Frankfurt, Germany
}

\begin{abstract}
We show that time correlation between electrons requires that the Dyson time ordering operator, $T$, differs from its uncorrelated value and spatial electron-electron correlation be present. In this paper we decompose $T$ into an uncorrelated term, $T_{u n c}$, plus a correlated term, $T_{\text {cor }}=T-T_{u n c}$, which leads to time correlation in time dependent external interactions. Effects of time correlation between electrons can be observed. Two examples are presented. In transfer ionization the time correlation operator incoherently changes the shape of an electron-electron Thomas peak. In double excitation the influence of $T_{\text {cor }}$ in amplitudes for coherently interfering pathways changes resonance intensities and profiles.
\end{abstract}

Understanding how electrons communicate about time requires ideas about both correlation and time. The mechanism for electrons to interact is the electron-electron Coulomb interaction, which is the source of spatial electron correlation (1). Without this spatial correlation the electrons are independent and cannot communicate. Time is often regarded as a parameter common to both the Schrödingerwave and Newtonian-particle equations. However, the way in which time operates is quite different in the wave and particle limits. In the quantum wave limit of broad delocalized wavepackets, operators for time are difficult to define (2), as reflected in Pauli's remark (3) that it is "impossible to find a self adjoint (local) time operator conjugate to any Hamiltonian with a bound spectrum (such as an atom)". Fortunately the mathematics of quantum mechanics is straightforward. The concept of time correlation has been used in non-equilibrium statistical quantum mechanics, where it is similar to spatial correlation $(4,5)$.

In this paper for the first time we specifically address the question of how time correlation between electrons affects cross sections for two electron transitions. The key conceptual tools of this paper are temporal correlation of the external interactions and spatial correlation between electrons. Both are required for time correlation between electrons. We give two examples in which time correlation between electrons plays an observable role in atomic reaction cross sections. The first case is a kinematic peak in a reaction in which electron transfer and ionization both occur. In this case time correlated and time uncorrelated amplitudes add incoherently. The second case is double electron excitation, where coherent reaction pathways interfere. In the second case time correlation between electrons produces a large observable effect on both the shape and intensity of a double excitation resonance.

In general, time correlation in many-body systems is basic to understanding timing among subsystems, cause and effect, dynamic control, and information processing. Transmission of information in multielectron quantum systems depends on how electrons are correlated in time. Control of reaction pathways in chemical and biological reactions $(6,7,8)$, application of fast atomic switching (9), time dependence in multi-electron quantum computing and quantum communication $(10,11)$ and in general dynamics of nanostructures $(12,13,14)$ all rely on understanding time correlation in multi-electron systems.

Time dependence is imposed on a quantum system $(2,15)$ by an external time dependent interaction, $V_{I}(t)$. The general expression for the probability amplitude, $a_{f i}(t)=<f\left|U_{I}\left(t, t_{i}\right)\right| i>$, for the transition of one or more electrons from $\mid i>$ at time $t_{i}$ to $\mid f>$ at time $t$ may be described most conveniently in the interaction representation $(5,16)$ using the evolution operator, $U_{I}\left(t, t^{\prime}\right)$, which satisfies,

$$
i \partial U_{I}\left(t, t^{\prime}\right) / \partial t=V_{I}(t) U_{I}\left(t, t^{\prime}\right)
$$


with the initial condition $\lim _{t \rightarrow-\infty} U_{I}(t,-\infty)=\hat{I}$. The formal solution for the evolution operator may be expressed as a time ordered exponential $(16,17)$,

$$
\begin{aligned}
& U_{I}\left(t, t_{i}\right)=T \exp \left\{-i \int_{t_{i}}^{t} V_{I}\left(t^{\prime}\right) d t^{\prime}\right\}= \\
& \sum_{k=0}^{\infty} \frac{(-i)^{k}}{k !} \int_{t_{i}}^{t} \ldots \int_{t_{i}}^{t} T\left[V_{I}\left(t_{1}\right) \ldots V_{I}\left(t_{k}\right)\right] d t_{1} \ldots d t_{k},
\end{aligned}
$$

where $T$ is the Dyson time ordering operator,

$$
\begin{aligned}
& T\left[V_{I}\left(t_{1}\right) V_{I}\left(t_{2}\right) \ldots V_{I}\left(t_{k}\right)\right] \\
& . \equiv \sum_{P(1,2, \ldots, k)} \theta\left(t_{1}-t_{2}\right) \theta\left(t_{2}-t_{3}\right) \ldots \theta\left(t_{k-1}-t_{k}\right) \\
& \times V_{I}\left(t_{1}\right) V_{I}\left(t_{2}\right) \ldots V_{I}\left(t_{k}\right) .
\end{aligned}
$$

Here $\theta\left(t-t^{\prime}\right)$ is the Heavyside step function. The sum above is taken over all possible permutations, $P$, of the parameters $1,2, \ldots, k$. The Dyson time ordering operator, $T$, imposes ordering of the $V_{I}\left(t_{j}\right)$ interactions in time to enforce causality in the time evolution of the system (16). Here $V_{I}(t)=\sum_{j}^{N} V_{I j}(t)$ is implicitly summed over electrons.

We seek correlation in time between the $V_{I}\left(t_{j}\right)$ 's, which provide (5) the time dependence to the quantum wave amplitudes, $a_{f i}(t)$, via Eq.(2). Requiring that correlation in time be independent of the mathematical form of $V_{I}(t)$, we use the only time dependent term available other than $V_{I}$, namely the time ordering operator, $T$. All time dependence in $T$ arises from the $\theta\left(t_{i}-t_{j}\right)$ terms in Eq.(3). Thus, time correlation may be removed by replacing all $\theta\left(t_{i}-t_{j}\right)$ by a constant. Then $T\left[V_{I}\left(t_{1}\right) V_{I}\left(t_{2}\right) \ldots V_{I}\left(t_{k}\right)\right]$ is a simple product of $V_{I}\left(t_{j}\right)$ and is therefore uncorrelated in time. Hence there can be no time correlation in $U_{I}$. Therefore, we now separate the $T$ operator into two terms,

$$
T=T_{\text {unc }}+\left(T-T_{\text {unc }}\right) \equiv T_{\text {unc }}+T_{\text {cor }} .
$$

where $T_{\text {unc }}$ is the uncorrelated part of $T$, and $T_{\text {cor }} \equiv$ $T-T_{u n c}$, acting on $V_{I}\left(t_{1}\right) \ldots V_{I}\left(t_{k}\right)$, is our time correlation operator. In first order in $V_{I}$ there is no time correlation. In second order one has,

$$
\begin{array}{r}
T\left(V_{I}(t) V_{I}\left(t^{\prime}\right)\right)=\theta\left(t-t^{\prime}\right) V_{I}(t) V_{I}\left(t^{\prime}\right) \\
+\theta\left(t^{\prime}-t\right) V_{I}\left(t^{\prime}\right) V_{I}(t)
\end{array}
$$

where,

$$
T_{u n c}\left(V_{I}(t) V_{I}\left(t^{\prime}\right)\right)=\frac{1}{2}\left(V_{I}(t) V_{I}\left(t^{\prime}\right)+V_{I}\left(t^{\prime}\right) V_{I}(t)\right)
$$

whence it is easily shown that,

$$
T_{\text {cor }}\left(V_{I}(t) V_{I}\left(t^{\prime}\right)\right)=\frac{1}{2} \operatorname{sign}\left(t-t^{\prime}\right)\left[V_{I}(t), V_{I}\left(t^{\prime}\right)\right] .
$$

Calculations using $T \simeq T_{\text {unc }}$ correspond to an independent time approximation (18), where the $V_{I}\left(t_{j}\right)$ interactions are not correlated in time. In second order a two step process is reduced to two independent one step processes $(19,20)$.

Since entanglement is conceptually and mathematically similar to electron correlation $(5,21)$, our time correlation operator, $T_{\text {cor }}$, may also be regarded as a time entanglement operator. Observable effects due to $T_{\text {cor }}$ occur in both single $(22,23)$ and multiple electron transitions (5). However, in this paper we consider only the effects of time correlation between electrons, i.e. how electrons communicate with one another about time.

In multiple electron transitions correlation in time between electrons generally requires spatial electronelectron correlation in addition to time ordering $(5,24)$. Physically this is obvious. In the uncorrelated independent electron approximation without exchange, the probability is represented as a product of single electron probabilities, namely, $P(t)=$ $\left|a_{f i}(t)\right|^{2}=\Pi_{j}\left|<f_{j}\right| U_{I j}\left(t, t_{i}\right)\left|i_{j}>\right|^{2}=\Pi_{j} P_{j}(t)$. In this limit there is no mechanism for time correlation between transitions of different electrons. Without spatial electron correlation phase information between electrons is lost. Only when spatial electron correlation is included can $T_{c o r}$ cause time correlation between different electron transition amplitudes.

In calculations presented in this paper electron exchange is included. Nevertheless, we note that it is conceptually convenient to neglect exchange. This simplifies the meaning of 'an electron' and 'an electron transition' and also it allows one to regard electrons as distinguishable. Inclusion of exchange is mathematically straightforward, but adds complexity both conceptually and technically. In fast atomic collisions the effects of exchange are often small.

In two examples below we have evaluated the effects of the $T_{\text {cor }}$ operator in calculations through second order in $V_{I}(t)$ by separating the second order term in $U_{I}$ into parts corresponding to the $T_{\text {unc }}$ and $T_{\text {cor }}$ parts of $T$. Calculations of cross sections are done with and without the $T_{c o r}$ time correlation terms.

As a first example let us consider a resonant reaction in which both electron transfer and ionization occurs, namely the purely second order electronelectron Thomas peak in transfer ionization (25). In this two step example a positively charged particle first interacts with an electron in an atomic target. Then the target electron rescatters from a second target electron such that it travels out of the collision with the projectile. Because of the presence of electron correlation the probability for this reaction 
cannot be written as a product of two independent electron transitions (transfer and ionization). The cross section for this peak is shown in figure 1. The effect of sequencing of the two interactions is carried by the $T_{\text {cor }}$ term in $U_{I}$. The effects of $T_{\text {unc }}$ and of the time correlation operator $T_{\text {cor }}$ add incoherently since the corresponding matrix elements differ by a factor of $i$ (27). The node in the contribution from time correlation at the center of the resonance is typical of anomalous dispersion (26), which connects the correlated contribution to the uncorrelated contribution and forces the correlated contribution to zero at the center of our resonance. This peak has been studied in detail experimentally $(28,29,30)$.

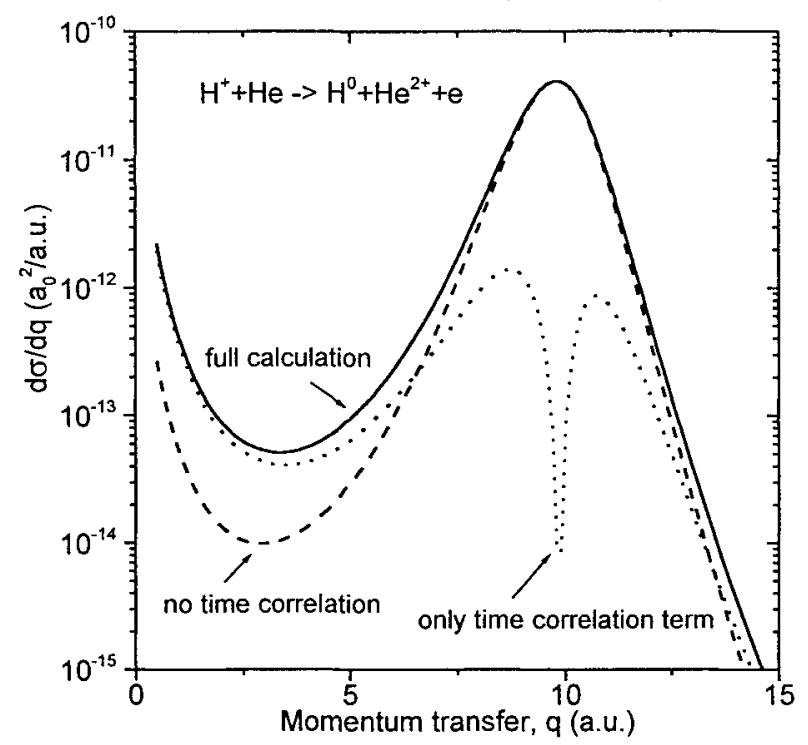

FIGURE 1. Cross section for transfer ionization as a function of the momentum transfer, $\mathrm{q}$, in $2.5 \mathrm{MeV}$ proton helium collisions in the vicinity of the electron-electron Thomas peak showing the effects of time correlation. Full curve, full second order calculation including both $T_{\text {cor }}$ and $T_{\text {unc }}$ terms of Eq.(4); long dash, approximate calculation using only the uncorrelated time term, $T_{u n c} ;$ short dash, approximate calculation using only the correlated time term, $T_{\text {cor }}$. In this case the effects of $T_{\text {cor }}$ and $T_{\text {unc }}$ add incoherently as explained in the text.

A second example is double electron excitation (31). In figure 2 we present calculations of the electron emission spectrum in the region of the $\left(2 p^{2}\right)^{1} D$ and $(2 s 2 p)^{1} P$ resonances of helium excited by 200 $\mathrm{eV}$ electron impact. Unlike the previous example, there is interference between reaction pathways in this case, namely direct single ionization and single ionization proceeding through the double excitation resonance. The effect of time correlation is amplified when the relative phase between competing pathways is close to $(2 n+1) \pi$. In figure 2 one sees a strong effect from the time correlation term on both the shape and the intensity of $\left(2 p^{2}\right)^{1} D$ and $(2 s 2 p)^{1} P$ resonance spectrum. In the $\left(2 p^{2}\right)^{1} D$ resonance time correlation changes the resonance shape from a window-type to a nearly asymmetric resonance profile. At the same time the intensity of the $(2 s 2 p)^{1} P$ resonance increases by a factor of three. The effect of time correlation varies with both scattering angle, $\theta_{f}$, and emission angle, $\theta_{e}$. Calculations for double electron excitation by fast ion impact also show clear effects due to time correlation. These resonances have been studied experimentally using high-resolution spectroscopy for both electron (32) and ion (31) impact.

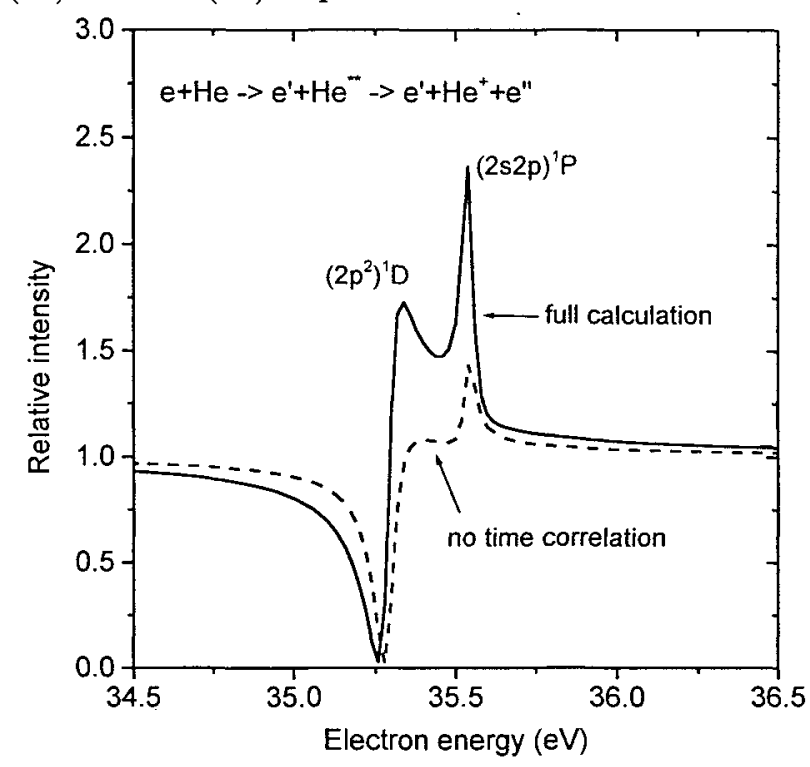

FIGURE 2. Effect of time-ordering on the autoionizing $\left(2 p^{2}\right)^{1} D$ and $(2 s 2 p)^{1} P$ resonances of helium in electron emission spectrum excited by $200 \mathrm{eV}$ electron impact. The electron angle of emission is $60^{\circ}$ and the projectile scattering angle is $30^{\circ}$. Full curve, full second order calculation including the $T_{\text {cor }}$ term of Eq. (4); broken curve, approximate calculation using only the uncorrelated time term, $T_{u n c}$. In this case the effects of $T_{\text {cor }}$ and $T_{u n c}$ are partially coherent. The cross section is normalized to the background of direct ionization.

In summary, we have considered time correlation between electrons in fast two electron transitions. Time dependence enters via an external $V_{I}(t)$. Time correlation among the $V_{I}\left(t_{j}\right)$ 's in the time evolution of the system is carried by the Dyson time ordering operator, $T$, which may be decomposed into an uncorrelated term, $T_{\text {unc }}$, plus a time correlation term, $T_{\text {cor }}=T-T_{\text {unc }}$. Interaction between electrons occurs via the two-body electron-electron correlation interactions, i.e., $1 / r_{i j}$ Coulomb interactions, sometimes modified by mean field potentials. This inter- 
action produces spatial electron correlation. When correlation in time is combined with spatial correlation, electrons are connected in time, as well as in space. This gives time correlation between electrons, which governs time sequencing in multielectron quantum systems. Effects of time correlation (entanglement in time) are observable. Two examples were given, one with and one without interfering pathways to a final state. Our approach applies to impact of ions, electrons and photons (including multi-photon effects) on atoms. Extension past second order in $V_{I}$, and also to more complex (e.g., nanoscale) systems, both appear feasible.

We thank P. Ivanov, V. Mergel, B. Shore and A. Goodman for stimulating discussions. This work was supported by the Division of Chemical Sciences, Office of Science, U.S. Department of Energy. R.D. acknowledges support by the Heisenberg Program der DFG.

\section{REFERENCES}

1. C. Froese Fischer, Atomic, Molecular and Optical Physics Reference Book, (G.W.F. Drake, ed.), AIP Press, NY, Chapter 21, (1996).

2. J.S. Briggs and J.M. Rost, Eur. Phys. J. D 10, 311 (2000); M. Murao, M.B. Plenio, S. Popescu, V. Vendral and P.L. Knight, Phys. Rev. A 57, R4075 (1999).

3. W. Pauli, Encyclopedia of Physics, edited by S. Flugge, Vol. 5/1, 60 (Springer, Berlin, 1958);

4. R. Balescu, Equilibrium and non-equilibrium Statistical Mechanics, John Wiley, NY, 1975), Chap. 21, Sec. 1. A simple product form is uncorrelated with this definition of correlation.

5. J.H. McGuire, Electron Correlation Dynamics in Atomic Collisions, (Cambridge University Press, 1997).

6. C. Winstead and V. McKoy, Adv. At., Mol., Opt. Phys. 43, in press.

7. L.R. LeClair and J.W. McConkey, J. Phys. B 27, 4039 (1995).

8. L.J. Dubé and P. Després, The Physics of Electronic and Atomic Collisions, ed. Y. Itikawa, AIP Conf. Proceedings, March 2000.

9. S. Gao, M. Persson, and B.I. Lundqvist, Solid State Communication 84, 271 (1992). (1993).

10. C.H. Bennett, D.P. DiVincenzo, J.A. Smolin and W.K. Wootten, Phys. Rev. A 54, 3824 (1996).

11. Colin P. Williams, Quantum Computing and Quantum Communications, (Springer, NY, 1999).
12. E.J. Heller, M.F. Crommie, C.P. Lutz and D.M. Eigler, Invited Talks of ICPEAC XIX, Whistler, Canada, AIP Conf. Proc. 360, 3 (1995).

13. J. Gao and J.B. Delos, Phys. Rev. A 56, 356 (1997).

14. M.T. Frey, F.B. Dunning, C.O. Reinhold, S. Yoshida, and J. Burgdörfer, Phys. Rev. A 59, 1434 (1999).

15. J.H. McGuire and O.L. Weaver, Phys. Rev. A34, 2473 (1986).

16. M.L. Goldberger and K.M. Watson Collision Theory, (Wiley, NY, 1964).

17. A.L. Fetter and J.D. Walecka, Quantum Theory of Many-Particle Systems. McGraw Hill, San Francisco, CA, (1971).

18. A.L. Godunov et al., in preparation.

19. G.R. Satchler, Direct Nuclear Reactions, (Oxford University Press, 1983), p. 300.

20. P.K. Bibdak and R.D. Koshel, Phys. Rev. C 6, 506 (1972).

21. R. Grobe, K. Rzazewski and J.H. Eberly, J. Phys. B. 27, L503 (1994).

22. H.Z. Zhao, Z.H. Lu and J.E. Thomas, Phys. Rev. Let. 79, 613 (1997).

23. L. Mandel and E. Wolf, Optical Coherence and Quantum Optics, (Cambridge University Press, 1995), Sections 4.3.1, 4.6.3 and 8.2.

24. L. Nagy, J.H.McGuire, L. Vegh, B. Sulik, and N. Stolterfoht, J. Phys. B 30, 1939 (1997); N. Stolterfoht, Phys. Rev. A 48, 2980 (1993).

25. S.G. Tolmanov and J.H. McGuire, Phys. Rev. A, 62, $032711(2000)$

26. J. H. McGuire and O. L. Weaver, J. Phys. B17, L583 (1984).

27. J.H. McGuire, A.L.Godunov, S.G.Tolmanov, H.Schmidt-Böcking, R.Dörner, V.Mergel, R.Dreizler and B.W.Shore, Intl. J. Mass Spectrometry 192, 65 (1999).

28. J. Palinkas et al., R. Schuch, H. Cederquist and O. Gustafsson, Phys. Rev. Let. 22, 2464 (1989).

29. V. Mergel, R. Dörner, M. Achler, Kh. Khayyat, S. Lencinas, J. Euler, O. Jagutzki, S. Nüttgens, M. Unverzagt, L. Spielberger, W. Wu, R. Ali, J. Ullrich, H. Cederquist, A. Salin, C.J. Wood, R.E. Olson, Dż. Belkić, C.L. Cocke, and H. Schmidt-Böcking, Phys. Rev. Let. 79, 387 (1997); V. Mergel, Ph.D thesis, Universität Frankfurt (1996).

30. R. Schuch, private communication.

31. A.L. Godunov, V. A. Schipakov, P. MorettoCapelle, D. Bordenave-Montesquieu, M. Benhenni, A. Bordenave-Montesquieu J. Phys. B: At. Mol. Opt. Phys., 30, 5451-5477 (1997).

32. J. Lower and E. Weigold, J. Phys. B: At. Mol. Opt. Phys., 23 2819-2845 (1990) 